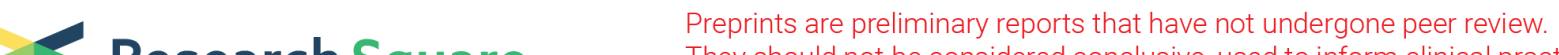 Research Square They should not be considered conclusive, used to inform clinical practice, or referenced by the media as validated information.
}

\section{Comparison of the Value of SOFA and Pitt Bacteremia Scores in the Prognostic Evaluation of Patients With Hospital-acquired Klebsiella Pneumoniae Bloodstream Infection}

Jun Guo ( $\sim$ junguo_med@tsinghua.edu.cn )

Beijing Tsinghua Changgung Hospital https://orcid.org/0000-0002-7374-2465

Shuaihua Fan

Tsinghua University School of Medicine

Jinlan Lin

Tsinghua University Affiliated Beijing Tsinghua Changgung Hospital: Beijing Tsinghua Changgung Hospital

Sheng Wu

Tsinghua University Affiliated Beijing Tsinghua Changgung Hospital: Beijing Tsinghua Changgung Hospital

\section{Research}

Keywords: SOFA score, Pitt bacteremia score, Klebsiella pneumoniae, bloodstream infection, Prognostic evaluation

Posted Date: October 11th, 2021

DOI: https://doi.org/10.21203/rs.3.rs-954898/v1

License: @ (i) This work is licensed under a Creative Commons Attribution 4.0 International License. Read Full License 


\section{Abstract}

This clinical research studied the value of SOFA score and Pitt bacteremia score in the prognosis assessment of patients with hospital-acquired Klebsiella pneumonia bloodstream infection. We conducted a retrospective analysis of 40 patients with hospital-acquired Klebsiella pneumoniae bloodstream infection in a tertiary hospital from January 2016 to December 2020. For these patients, the SOFA score and Pitt bacteremia score were used to evaluate the prognosis. Logistic regression was performed with the known prognosis results to obtain the best cut-off value, sensitivity, and specificity. Pitt bacteremia score [3 (3-4) points to 6 (5.5-7) points] and SOFA score [7 (6-10) points to 17 (13-17.5) points] in the survival group were lower than those in the death group $(P<0.05)$. The SOFA score predicts the death of hospital-acquired Klebsiella pneumoniae bloodstream infection patients with a sensitivity of $80 \%$, a specificity of $84 \%$, and the area under curve(AUC) of SOFA score is 0.8960 ( $95 \% \mathrm{Cl} 0.7951-0.9969)$; Pitt bacteremia score predicts the hospital-acquired Klebsiella pneumoniae blood infection with a sensitivity of $86.67 \%$, a specificity of $80 \%$, and AUC of Pitt bacteremia score is 0.9413 ( $95 \% \mathrm{Cl} 0.8700$ 1.000). Both the SOFA score and the Pitt bacteremia score have predictive value for the prognosis of patients with HAl (hospital acquired infection) Klebsiella pneumonia blood infection. However, the difference shows that the SOFA score has obvious accuracy and specificity in the prognosis of patients with HAI Klebsiella pneumoniae bloodstream infection, it is better than the Pitt bacteremia score and has greater application prospects in prognostic evaluation.

\section{Introduction}

As the Centers for Disease Control and Prevention (CDC) identified, complications or infections secondary to device implantation or surgery are called $\mathrm{HAl}^{(1)}$. Tens of thousands of people are infected worldwide every year ${ }^{(2,3)}$. According to statistics data, about 2 million patients suffer from HAl each year in the United States with 99,000 total deaths, which costs 33 billion dollars each year ${ }^{(4,5)}$. In European, the number of people who die directly from HAl each year is about 37,000, and the total length of hospital stay increases by 1,600 Million $^{(6)}$. Bloodstream infection is one of the important infection types in HAI. Musicha $\mathrm{P}$ et al ${ }^{(7)}$ showed that the mortality rate of bloodstream infection in HAl is higher, and the incidence of Klebsiella pneumoniae bloodstream infection in Enterobacteriaceae is in the forefront. CHINET(China Antimicrobial Surveillance Network) shows the isolation rate of Klebsiella pneumoniae bloodstream infection is $16.51 \%$, which is the second place among bloodstream infection bacteria (8). What's worse is that the resistance rate of Klebsiella pneumoniae is increasing every year. According to calculations, 426,277 cases of HAl caused by antimicrobial drug-resistant microorganisms occur in the EU every year, and the number of deaths due to drug-resistant microorganisms in the $\mathrm{EU}$ is 33,110 each

year ${ }^{(9)}$. Therefore, the early assessment for the condition of patients with Klebsiella pneumoniae infection can effectively guide the selection of clinical treatment and the intensity of care levels, as to minimize the mortality rate and improve the prognosis. Then the criteria for evaluating the prognosis of the disease are particularly important. After continuous clinical practice, many scholars have formed a scoring system 
used in a variety of different scenarios. The Pitt bacteremia score and the SOFA score are currently two widely used clinical scoring system.

The Pitt bacteremia score was first proposed by Rasmussen $\mathrm{HH}$ et al ${ }^{(10)}$ in 1985 . Its scoring items include temperature, blood pressure, mechanical ventilation, cardiopulmonary resuscitation, and mental status. The total score is 18 points. The higher the score, the worse the prognosis. Recently, many studies confirmed that the Pitt bacteremia score has great significance for the prognosis assessment of acute and critical illness ${ }^{(11,12)}$, and many studies have shown that Pitt bacteremia score $>4$ can be used as a risk factor for death $(13,14)$, so it is widely recognized by domestic and foreign researchers and clinicians to evaluate the prognosis of critical patients. The SOFA score was first proposed in 1994 by the European Society of Intensive Care Medicine ${ }^{(15)}$, and its purpose is to describe the occurrence, development and incidence of MODS. It includes the assessment of the respiratory system, coagulation system, liver, circulatory system, nervous system and kidneys. The total score is 40 points. The higher the score, the worse the prognosis. A number of studies have shown that SOFA scores can be used to evaluate the prognosis of critically ill patients ${ }^{(16,17)}$, so it is widely used in clinical evaluation of the prognosis of critically ill patients. However, there are still no relevant studies on the evaluation of the Pitt bacteremia score and SOFA score in the prognosis of patients with bloodborne infections.

This study retrospectively analyzed the prognostic evaluation of SOFA score and Pitt bacteremia score in 40 patients with hospital acquired Klebsiella pneumoniae bloodstream infection. The objective has explored the application value and advantages and disadvantages of SOFA score and Pitt bacteremia score in the prognosis evaluation of HAI patients with Klebsiella pneumoniae bloodstream infection.

\section{Research Object, Clinical Diagnosis And Methods 2.1 Research object}

A retrospective analysis of $\mathrm{HAl}$ (hospital acquired infection) cases was conducted in a tertiary hospital which has a total 1000 beds in Beijing, China. From January 2016 to December 2020, a total of 1879 cases of HAl were reported, including 225 cases of bloodstream infection, 54 cases of Klebsiella pneumoniae blood infection, except for 14 patients with incomplete case data. A total of 40 cases of HAI Klebsiella pneumoniae blood infection were included in this study. In this study, a special CRF table was designed, and Pitt bacteremia score and SOFA score were calculated on these 40 patients based on medical records, examination and test results.

Inclusion criteria: (1) According to the definition, it was identified as a HAl patient with complete medical records, examination and test results, and clear prognostic information; (2) Strain isolation and culture of clinical specimens according to the National Clinical Laboratory Procedures"(18), The Mérieux Vitek-2 Compact automatic microbial system was used to identify the bacterial species, and the blood culture microbiological examination and drug sensitivity test indicated that it was Klebsiella pneumoniae infection; (3) There were at least 2 blood culture results. 
Exclusion criteria: (1) Patients with incomplete clinical data such as medical records, examinations, and tests; (2) Suspected blood samples are contaminated, and common skin colonization bacteria isolated from a single bottle of blood culture are inconsistent with clinical symptoms and signs, and can be other Reasons explained; (3) Only one blood culture result.

\subsection{Clinical diagnosis}

1. HAI Klebsiella pneumoniae infection: During the hospitalization period, complete blood culture examinations and drug susceptibility tests are confirmed to be Klebsiella pneumoniae, except that the sample may be contaminated, and the HAI Klebsiella pneumonia infection can be diagnosed.

2. Blood infection: There are related manifestations of blood infection: chills, high fever, tachycardia, shortness of breath, skin rash, mental and mental changes, etc.

3. Etiology: There are relevant etiology and bacterial culture evidence to verify.

\subsection{Methods}

Graphpad Prism8 statistical software was used for data analysis. 1. Count variables are represented by median and interquartile range. 2. As a grade variables, the results of Pitt bacteremia score and SOFA score were convert to dichotomous variables by Logistic regression. (1) Perform Logistic regression on the scores of the 40 groups and the prognosis to obtain the best cut-off value, and use the Youden Index (a method to evaluate the authenticity of the screening test, which is the sum of sensitivity and specificity minus 1) to verify the cut-off value accuracy, and then perform ROC curve analysis separately with the prognostic quantitative variables, and evaluate the prognosis of the two scores by comparing the area under the curve; (2) The cut-off value was used to determine the positive and negative predictive results. The sensitivity, specificity, positive predictive value and negative predictive value of the two groups were compared to evaluate the predictive effect of the two scores on the prognosis. (3) Retrospectively analyze the predicted survival time and actual survival time of 40 patients, and draw a survival curve. Comparing with the known prognosis results, it is more intuitive to reflect the accuracy of the two scores in evaluating the prognosis of such patients.

\section{Results}

\subsection{Analysis of the mortality of the study population}

The brief information of all the included cases is shown below: 
Table 1

Basic information of the enrolled cases

\begin{tabular}{|c|c|c|c|c|c|c|c|c|}
\hline $\begin{array}{l}\text { Serial } \\
\text { number }\end{array}$ & Age & Gender & $\begin{array}{l}\text { Pitt } \\
\text { bacteremia } \\
\text { score }\end{array}$ & $\begin{array}{l}\text { SOFA } \\
\text { score }\end{array}$ & $\begin{array}{l}\text { Gastric } \\
\text { tube }\end{array}$ & $\begin{array}{l}\text { Urinary } \\
\text { tube }\end{array}$ & $\begin{array}{l}\text { Mechanical } \\
\text { ventilation }\end{array}$ & $\begin{array}{l}\text { Survival } \\
\text { situation }\end{array}$ \\
\hline 1 & 59 & Female & 4 & 7 & No & Yes & Yes & Died \\
\hline 2 & 54 & Female & 3 & 9 & Yes & Yes & Yes & Survived \\
\hline 3 & 68 & Male & 4 & 10 & Yes & Yes & Yes & Survived \\
\hline 4 & 77 & Male & 4 & 18 & Yes & Yes & Yes & Died \\
\hline 5 & 52 & Male & 6 & 21 & Yes & Yes & Yes & Died \\
\hline 6 & 35 & Male & 2 & 6 & Yes & Yes & Yes & Survived \\
\hline 7 & 1 & Female & 7 & 15 & Yes & Yes & Yes & Died \\
\hline 8 & 54 & Male & 7 & 17 & Yes & Yes & Yes & Died \\
\hline 9 & 70 & Male & 5 & 17 & Yes & Yes & Yes & Died \\
\hline 10 & 82 & Male & 6 & 14 & Yes & Yes & Yes & Died \\
\hline 11 & 50 & Female & 3 & 5 & Yes & Yes & Yes & Survived \\
\hline 12 & 67 & Male & 4 & 7 & Yes & Yes & Yes & Survived \\
\hline 13 & 36 & Male & 6 & 14 & Yes & Yes & Yes & Survived \\
\hline 14 & 52 & Male & 6 & 13 & Yes & Yes & Yes & Survived \\
\hline 15 & 60 & Male & 5 & 13 & Yes & Yes & Yes & Survived \\
\hline 16 & 60 & Male & 5 & 13 & Yes & Yes & Yes & Survived \\
\hline 17 & 77 & Female & 7 & 12 & Yes & Yes & Yes & Survived \\
\hline 18 & 78 & Female & 6 & 12 & Yes & Yes & Yes & Survived \\
\hline 19 & 35 & Male & 4 & 12 & Yes & Yes & Yes & Survived \\
\hline 20 & 65 & Male & 4 & 12 & Yes & Yes & Yes & Died \\
\hline 21 & 61 & Male & 4 & 10 & Yes & Yes & Yes & Died \\
\hline 22 & 47 & Male & 5 & 9 & Yes & Yes & Yes & Survived \\
\hline 23 & 41 & Female & 3 & 9 & Yes & Yes & Yes & Survived \\
\hline 24 & 85 & Female & 3 & 9 & Yes & Yes & Yes & Survived \\
\hline 25 & 83 & Female & 3 & 9 & Yes & Yes & No & Survived \\
\hline
\end{tabular}




\begin{tabular}{|c|c|c|c|c|c|c|c|c|}
\hline $\begin{array}{l}\text { Serial } \\
\text { number }\end{array}$ & Age & Gender & $\begin{array}{l}\text { Pitt } \\
\text { bacteremia } \\
\text { score }\end{array}$ & $\begin{array}{l}\text { SOFA } \\
\text { score }\end{array}$ & $\begin{array}{l}\text { Gastric } \\
\text { tube }\end{array}$ & $\begin{array}{l}\text { Urinary } \\
\text { tube }\end{array}$ & $\begin{array}{l}\text { Mechanical } \\
\text { ventilation }\end{array}$ & $\begin{array}{l}\text { Survival } \\
\text { situation }\end{array}$ \\
\hline 26 & 51 & Male & 4 & 8 & Yes & Yes & No & Survived \\
\hline 27 & 56 & Male & 5 & 7 & Yes & Yes & Yes & Survived \\
\hline 28 & 85 & Male & 5 & 7 & Yes & Yes & Yes & Died \\
\hline 29 & 43 & Female & 4 & 7 & Yes & Yes & Yes & Died \\
\hline 30 & 65 & Male & 4 & 7 & Yes & Yes & Yes & Died \\
\hline 31 & 75 & Male & 4 & 7 & Yes & Yes & Yes & Died \\
\hline 32 & 70 & Male & 4 & 7 & Yes & Yes & No & Survived \\
\hline 33 & 17 & Male & 3 & 7 & Yes & Yes & No & Survived \\
\hline 34 & 34 & Male & 2 & 6 & Yes & Yes & No & Survived \\
\hline 35 & 84 & Male & 2 & 6 & Yes & Yes & Yes & Died \\
\hline 36 & 30 & Female & 3 & 5 & Yes & Yes & Yes & Survived \\
\hline 37 & 38 & Female & 3 & 4 & Yes & Yes & No & Survived \\
\hline 38 & 19 & Male & 3 & 4 & Yes & No & No & Survived \\
\hline 39 & 73 & Female & 2 & 4 & Yes & Yes & Yes & Died \\
\hline 40 & 51 & Male & 3 & 2 & Yes & Yes & Yes & Survived \\
\hline
\end{tabular}

Table 2

Statistical description of enrollment

\begin{tabular}{|llll|}
\hline Characteristic & $\begin{array}{l}\text { Survived(Median [IQR] or } \\
\mathbf{n} \%) \mathbf{n = 2 5}\end{array}$ & $\begin{array}{l}\text { Died (Median [IQR] or } \\
\mathbf{n} \%) \mathbf{n = 1 5}\end{array}$ & $\begin{array}{l}\text { Total (Median [IQR] or } \\
\mathbf{n} \%) \mathbf{n = 4 0}\end{array}$ \\
\hline Age & $51(36-67)$ & $65(56.5-76)$ & $57.5(42.5-70.75)$ \\
\hline Male & $18(72)$ & $10(66.67)$ & $28(70)$ \\
\hline Pitt score & $3(3-4)$ & $6(5.5-7)$ & $4(3-6)$ \\
\hline SOFA score & $7(6-10)$ & $17(13-17.5)$ & $11(7-15)$ \\
\hline
\end{tabular}

In all 40 cases of Klebsiella pneumoniae hematological infections, the median age was 57.5 years (interquartile range 42.5-70.75), of which 51 (36-67) in the survival group and the death group was 65 
(56.5-76) (Table 1). The overall mortality rate of these hospitalized patients was $37.5 \%$. The Pitt bacteremia score of the survival group was 3 (3-4), the death group was 6 (5.5-7); the SOFA score of the survival group was 7 (6-10), and the death group was 17 (13-17.5).

\subsection{Selection and analysis of the best cut-off value}

The 40 groups of Pitt bacteremia score results and prognostic results ( 0 means survival, 1 means death) are used for Logistic regression, and the best cut-off value is 4 points. By calculation, when the cut-off value is 4 points, the Youden Index also reaches its maximum. Under the best cut-off value, the Pitt bacteremia score is calculated to have a sensitivity of $86.67 \%$, a specificity of $80 \%$, and a total prediction accuracy of $82.5 \%$ (Table 2) for predicting the death of patients with HAI Klebsiella pneumoniae infection. The negative predictive value was $90.91 \%$, and the positive predictive value was $72.22 \%$.

In the same way, using Logistic regression to analysis the obtained 40 groups of SOFA score results and prognostic results ( 0 means survival, 1 means death), the best cut-off value is 13 points. By calculation, when the cut-off value is 13 points, the Youden index also reached the maximum. Under the best cut-off value, the SOFA score has a sensitivity of $66.67 \%$, a specificity of $88 \%$, a total prediction accuracy of $80 \%$ (Table 3), the negative predictive value is $81.48 \%$, and the positive predictive value was $76.92 \%$.

Table 3

Predictive comparison of Pitt bacteremia score and SOFA score

\begin{tabular}{|llllll|}
\hline \multirow{3}{*}{ Pitt bacteremia score } & & Survived & Died & Total & \% Correctly classified \\
& Survived & 20 & 5 & 25 & 80 \\
\cline { 2 - 6 } & Died & 2 & 13 & 15 & 86.67 \\
\cline { 2 - 6 } & Total & 22 & 18 & 40 & 82.5 \\
\hline SOFA score & Survived & 22 & 3 & 25 & 88 \\
\cline { 2 - 6 } & Died & 5 & 10 & 15 & 66.67 \\
\hline Total & 27 & 13 & 40 & 80 \\
\hline
\end{tabular}

\subsection{The ratio of Pitt bacteremia score and SOFA score to the prediction of prognosis}

According to the best cut-off value obtained, the Pitt bacteremia score and SOFA score were made into ROC curves to be compared (Figure 3). According to the results of ROC analysis, the AUC of the Pitt bacteremia score was $0.9413(95 \% \mathrm{Cl} 0.8700-1.000)$, and the AUC of the SOFA score was $0.8960(95 \% \mathrm{Cl}$ 0.7951-0.9969).

\section{Discussion And Conclusion}


A number of studies have shown that HAl could cause the mortality, morbidity, length of stay (LOS) and economic burden of hospitalized patients to be too high(4, 19-22). The total hospitalization cost and length of stay of HAI patients are significantly longer than General patients ${ }^{(19,21,22)}$. In Chinese mainland, Klebsiella pneumoniae is the second common Gram-negative pathogen causing hospital bloodstream infection after E.coli(23-26). In recent years, with the use of broad-spectrum antibiotics in medical settings more and more common, drug-resistant Klebsiella pneumoniae has emerged and spread quickly, which has caused many problems for clinical practice. For this reason, the World Health Organization issued a global action plan for antimicrobial resistance in 2015, and take antibiotic management as the core pillar of this plan ${ }^{(27)}$. Therefore, it is important to predict the outcome of nosocomial infection early and correctly. The SOFA score mainly predicts the occurrence or development of critical illness by observing the dynamic changes of organ function, so as to accurately judge the condition; the Pitt bacteremia score quickly judges the development process of the patient's condition based on the patient's general condition.

In this study, both the Pitt bacteremia score and SOFA score were calculated respectively, the prognosis obtained from these two scores were compared with actual prognosis. Through epidemiological analysis (Table 2), it is found that the age of the survival group is slightly smaller than that of the death group; the Pitt bacteremia score and SOFA score of the survival group are significantly lower than those of the death group, which preliminarily shows that there was a certain correlation between these two scores and prognosis. On this basis, the best cut-off value was found through Logistic regression and found that Pitt bacteremia score $>4$ points and SOFA score $>13$ points are good predictors for the prognosis of patients with HAI Klebsiella pneumoniae bloodstream infection. The fit is relatively high. The Pitt bacteremia score ( $86.67 \%)$ is slightly more sensitive to prognostic evaluation than the SOFA score (66.67\%), but the specificity of the SOFA score (88\%) is better than the Pitt bacteremia score (80\%), The negative predictive value of Pitt bacteremia score was higher $(90.91 \%)$, and there may be the possibility of aggravating clinical condition. From the perspective of the survival curve (Figure 2), the SOFA score has better performance than the Pitt bacteremia score in evaluating the survival days of patients; from the perspective of the ROC curve (Figure 3), the fit of the Pitt bacteremia score for the prognostic evaluation is slightly better than the SOFA score. Overall, the SOFA score and Pitt bacteremia score both have good performance in the prognosis evaluation of patients with HAI Klebsiella pneumoniae bloodstream infection, but the accuracy and specificity of SOFA score is significantly better than Pitt bacteremia score, and it has greater prospects in prognostic evaluation.

In this retrospective study, we innovatively compared the Pitt bacteremia score and SOFA score for the prognosis of hospital acquired bloodstream infection caused by Klebsiella pneumoniae for the first time. The scientificity of the clinical application of Pitt bacteremia score and SOFA score is verified, and some references are provided for such research. At the same time, all patients in this study received the same medical resources, excluding the prognostic infection caused by the interference of the external environment, which improved the credibility of the results. However, since this study is a single-center retrospective study, the sample size is limited, and the available data are only medical records, 
examinations and test results kept in the hospital, and it may not be possible to objectively evaluate the patient's condition at time. Therefore, it is expected that studies with more complete designs and larger sample sizes will provide more accurate comparative data.

\section{Declarations}

This manuscript has been reviewed by the ethics committee of our hospital, and there is no potential ethical risk.

\section{Acknowledgements}

The authors thank the Inpatient information Management Department of Beijing Tsinghua Changgung Hospital for the help in data retrieval.

\section{Disclosure statement}

The authors declare no conflict of interest regarding the publication of this paper.

\section{Funding}

This study was not supported by any foundation.

\section{References}

1. CDC. 2016 2016. National and State Healthcare-Associated Infections progress report. 2016, on Centers for Disease Control and Prevention. http://www.cdc.gov/HAl/pdfs/progress-report/haiprogress-report. Accessed.

2. Allegranzi B, Bagheri Nejad S, Combescure C, Graafmans W, Attar H, Donaldson L, Pittet D. Burden of endemic health-care-associated infection in developing countries: systematic review and metaanalysis. Lancet. 2011;377:228-41.

3. Bagheri Nejad S, Allegranzi B, Syed SB, Ellis B, Pittet D. Health-care-associated infection in Africa: a systematic review. Bull World Health Organ. 2011;89:757-65.

4. Klevens RM, Edwards JR, Richards CL Jr, Horan TC, Gaynes RP, Pollock DA, Cardo DM. 2007. Estimating health care-associated infections and deaths in U.S. hospitals, 2002. Public Health Rep 122:160-6.

5. Scott RD. 2009 2009. The Direct MeDical costs of Healthcare-Associated Infections in U.S. Hospitals and the Benefits of Prevention, on Centers for Disease Control and prevention. http://www.cdc.gov/hai/pdfs/hai/scott_costpaper. Accessed.

6. Picture C. 2009. European Centre for Disease Prevention and Control. Annual Epidemiological Report on Communicable Diseases in Europe 2009. 
7. Musicha P, Cornick JE, Bar-Zeev N, French N, Masesa C, Denis B, Kennedy N, Mallewa J, Gordon MA, Msefula CL, Heyderman RS, Everett DB, Feasey NA. Trends in antimicrobial resistance in bloodstream infection isolates at a large urban hospital in Malawi (1998-2016): a surveillance study. Lancet Infect Dis. 2017;17:1042-52.

8. CHINET. 2020 2020. Distribution of main strains of 37,737 strains of blood specimen isolates in 2020., on CHINET. http://www.chinets.com/Data/AntibioticDrugFast. Accessed.

9. Cassini A, Hogberg LD, Plachouras D, Quattrocchi A, Hoxha A, Simonsen GS, Colomb-Cotinat M, Kretzschmar ME, Devleesschauwer B, Cecchini M, Ouakrim DA, Oliveira TC, Struelens MJ, Suetens C, Monnet DL. Burden of AMRCG. 2019. Attributable deaths and disability-adjusted life-years caused by infections with antibiotic-resistant bacteria in the EU and the European Economic Area in 2015: a population-level modelling analysis. Lancet Infect Dis 19:56-66.

10. Rasmussen HH, Pitt EA, Ibels LS, McNeil DR. Prediction of outcome in acute renal failure by discriminant analysis of clinical variables. Arch Intern Med. 1985;145:2015-8.

11. Al-Hasan MN, Baddour LM. Resilience of the Pitt Bacteremia Score: 3 Decades and Counting. Clin Infect Dis. 2020;70:1834-6.

12. Jorgensen SCJ, Trinh TD, Zasowski EJ, Lagnf AM, Bhatia S, Melvin SM, Simon SP, Rosenberg JR, Steed ME, Estrada SJ, Morrisette T, Davis SL, Rybak MJ. Evaluation of the INCREMENT-CPE, Pitt Bacteremia and qPitt Scores in Patients with Carbapenem-Resistant Enterobacteriaceae Infections Treated with Ceftazidime-Avibactam. Infect Dis Ther. 2020;9:291-304.

13. Henderson H, Luterbach CL, Cober E, Richter SS, Salata RA, Kalayjian RC, Watkins RR, Doi Y, Kaye KS, Evans S, Fowler VG, Bonomo RA, Harris A, Napravnik S, Van Duin D. The Pitt Bacteremia Score Predicts Mortality in Nonbacteremic Infections. Clin Infect Dis. 2020;70:1826-33.

14. Wu Z, Chen Y, Xiao T, Niu T, Shi Q, Xiao Y. Epidemiology and risk factors of infective endocarditis in a tertiary hospital in China from 2007 to 2016. BMC Infect Dis. 2020;20:428.

15. Pawar RD, Shih JA, Balaji L, Grossestreuer AV, Patel PV, Hansen CK, Donnino MW, Moskowitz A. Variation in SOFA (Sequential Organ Failure Assessment) Score Performance in Different Infectious States. J Intensive Care Med. 2020. doi:10.1177/0885066620944879:885066620944879.

16. Dickstein Y, Leibovici L, Yahav D, Eliakim-Raz N, Daikos GL, Skiada A, Antoniadou A, Carmeli Y, Nutman A, Levi I, Adler A, Durante-Mangoni E, Andini R, Cavezza G, Mouton JW, Wijma RA, Theuretzbacher U, Friberg LE, Kristoffersson AN, Zusman O, Koppel F, Dishon Benattar Y, Altunin S, Paul M, consortium A. Multicentre open-label randomised controlled trial to compare colistin alone with colistin plus meropenem for the treatment of severe infections caused by carbapenem-resistant Gram-negative infections (AIDA): a study protocol. BMJ Open. 2016;6:e009956.

17. Vincent JL, Moreno R, Takala J, Willatts S, De Mendonca A, Bruining H, Reinhart CK, Suter PM, Thijs LG. The SOFA (Sepsis-related Organ Failure Assessment) score to describe organ dysfunction/failure. On behalf of the Working Group on Sepsis-Related Problems of the European Society of Intensive Care Medicine. Intensive Care Med. 1996;22:707-10. 
18. Department of Medical Administration MoHotPsRoC. National Clinical Laboratory Procedures. 3rd ed. Nanjing: Southeast University Press; 2006.

19. Glance LG, Stone PW, Mukamel DB, Dick AW. Increases in mortality, length of stay, and cost associated with hospital-acquired infections in trauma patients. Arch Surg. 2011;146:794-801.

20. Graves N. Economics and preventing hospital-acquired infection. Emerg Infect Dis. 2004;10:561-6.

21. Jarvis WR. Selected aspects of the socioeconomic impact of nosocomial infections: morbidity, mortality, cost, and prevention. Infect Control Hosp Epidemiol. 1996;17:552-7.

22. Stone PW. Economic burden of healthcare-associated infections: an American perspective. Expert Rev Pharmacoecon Outcomes Res. 2009;9:417-22.

23. Jiang ZQ, Wang SD, Feng DD, Zhang BX, Mao SH, Wu JN. Epidemiological risk factors for nosocomial bloodstream infections: A four-year retrospective study in China. J Crit Care. 2019;52:92-6.

24. Tian L, Sun Z, Zhang Z. Antimicrobial resistance of pathogens causing nosocomial bloodstream infection in Hubei Province, China, from 2014 to 2016: a multicenter retrospective study. BMC Public Health. 2018;18:1121.

25. Wu JN, Gan TE, Zhu YX, Cao JM, Ji CH, Wu YH, Lv B. Epidemiology and microbiology of nosocomial bloodstream infections: analysis of 482 cases from a retrospective surveillance study. J Zhejiang Univ Sci B. 2015;16:70-7.

26. Xiao T, Yu W, Niu T, Huang C, Xiao Y. A retrospective, comparative analysis of risk factors and outcomes in carbapenem-susceptible and carbapenem-nonsusceptible Klebsiella pneumoniae bloodstream infections: tigecycline significantly increases the mortality. Infect Drug Resist. 2018;11:595-606.

27. WHO. 2015 2015. Global Action Plan on Antimicrobial Resistance., on WHO. https://apps.who.int/iris/handle/10665/193736. Accessed.

\section{Figures}




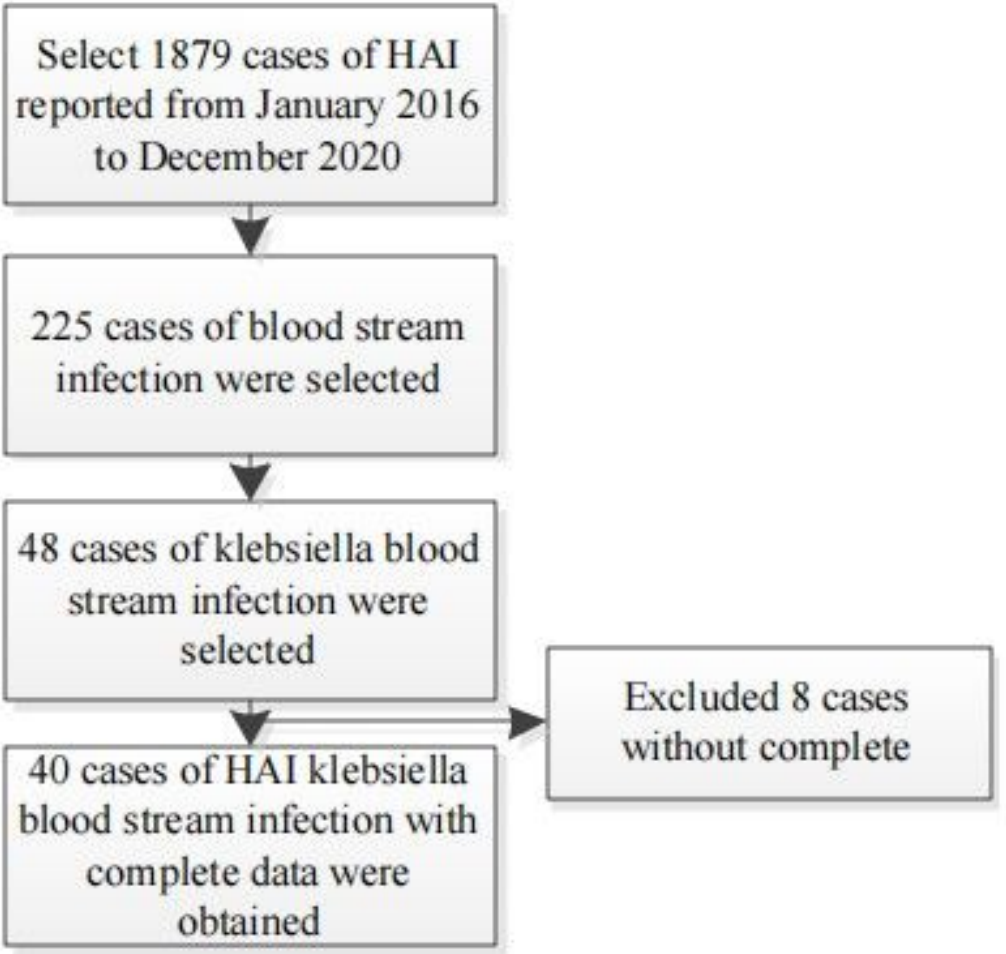

Figure 1

Selection of study

\section{SOFA Score}

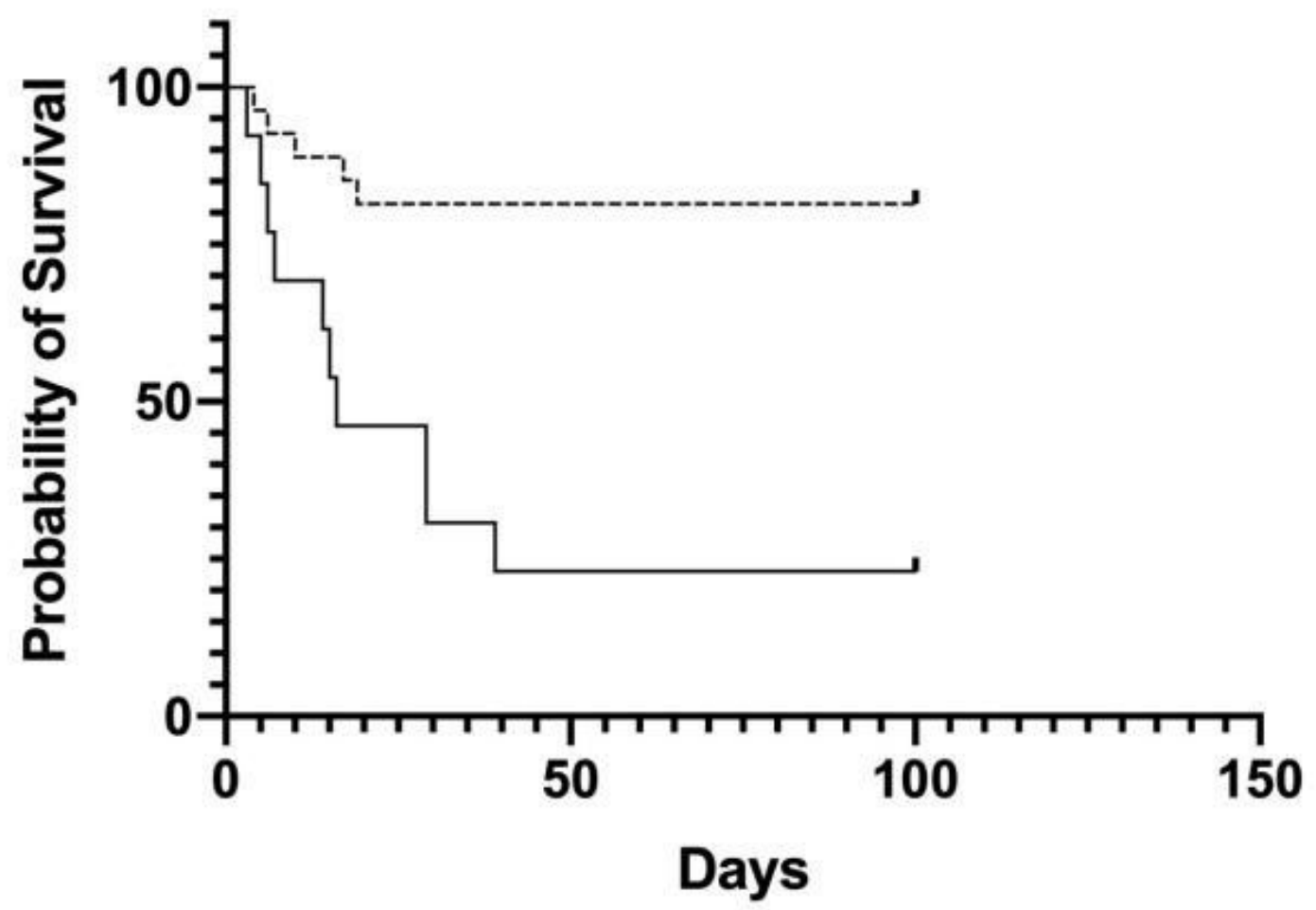




\section{Pitt Bacteremia Score}

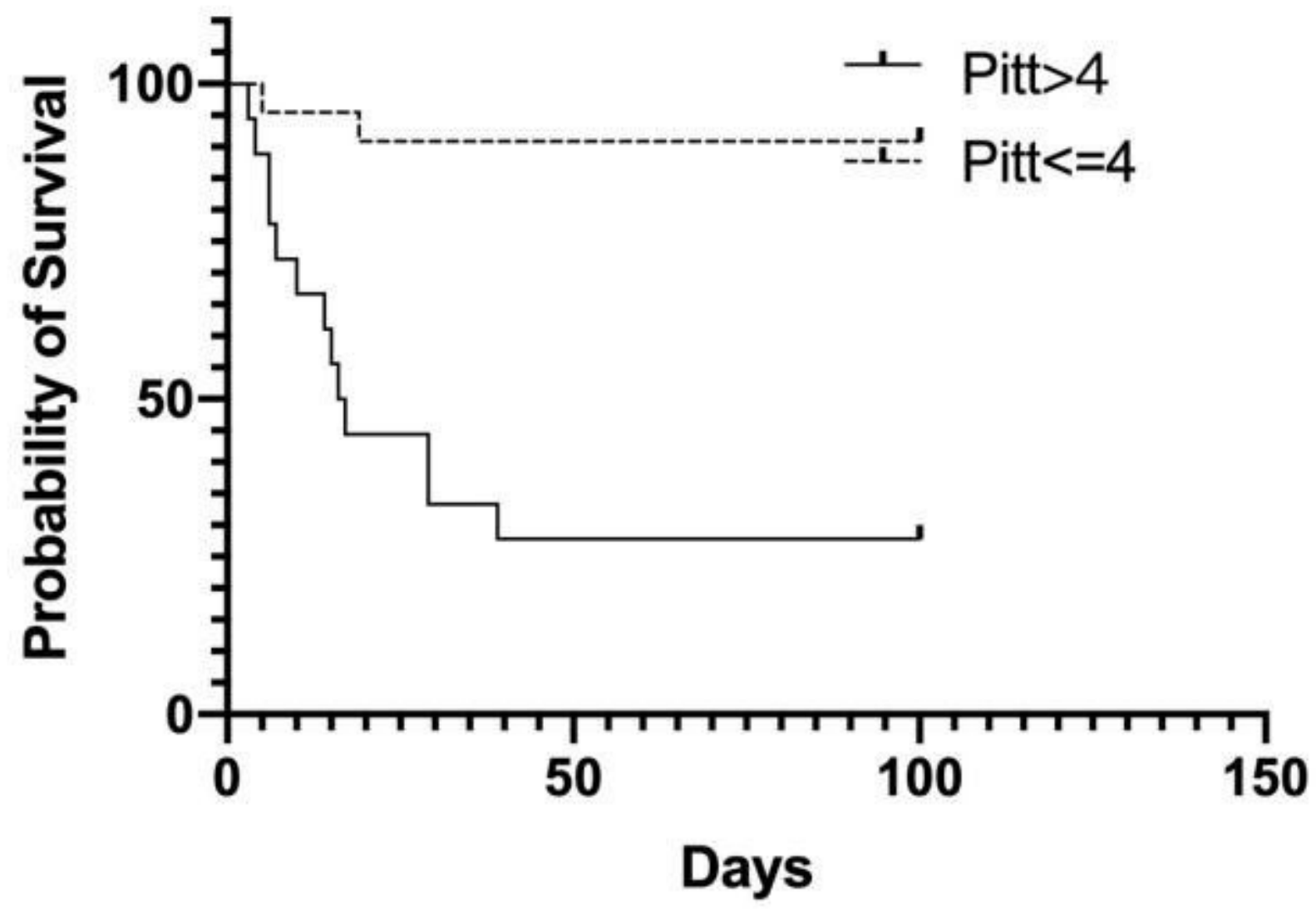

Figure 3

Survival rate curve of Pitt bacteremia score 


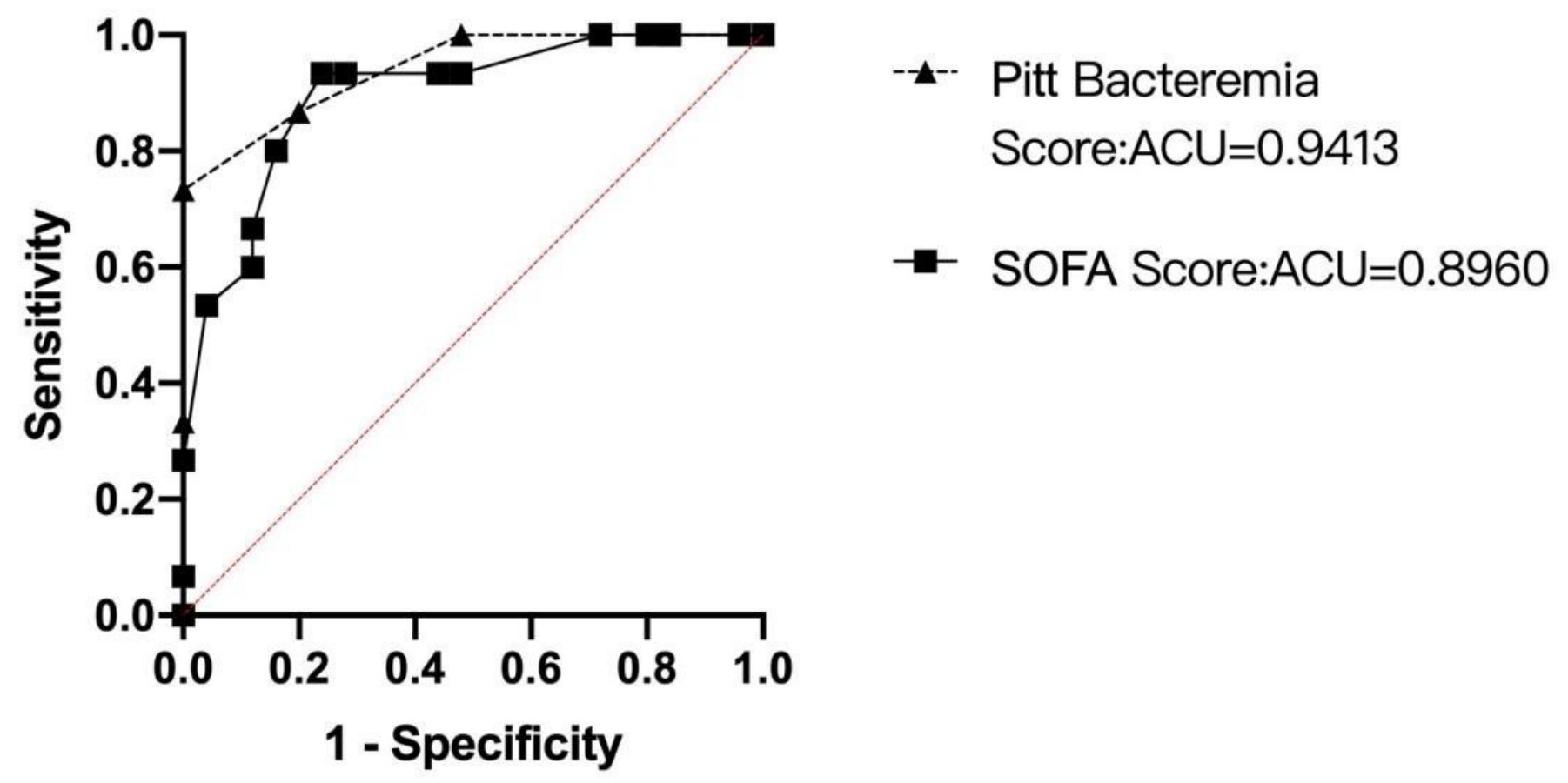

Figure 4

Comparison of Pitt bacteremia score and SOFA score 